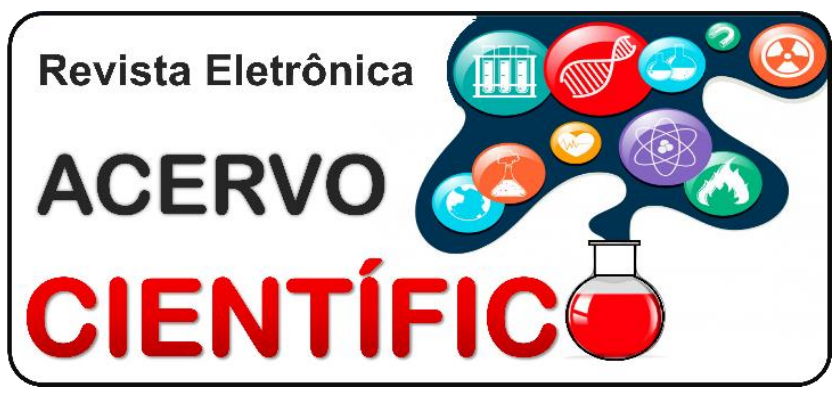

REVISÃO BIBLIOGRÁFICA

Recebido em: 10/2020

Aceito em: 12/2020

Publicado em: 2/2021

\title{
Abordagem cirúrgica convencional e percutânea para tratamento de Ostium Secundum: uma revisão da literatura
}

\author{
Conventional and percutaneous surgical approach for the treatment of Ostium Secundum: \\ a literature review
}

\begin{abstract}
Abordaje quirúrgico convencional y percutáneo para el tratamiento del Ostium Secundum: un repaso literario
\end{abstract}

Amanda Rodrigues Rios ${ }^{1 *}$, Aline Trovão Queiroz ${ }^{1}$, Camila Pereira Morbelli ${ }^{3}$, Gabriel de Souza Cabideli $^{3}$, Gabriela Monteiro Silva ${ }^{3}$, Gustavo Folz Rossini ${ }^{3}$, Julia Cazelli Passos Ricardo ${ }^{3}$, Juliana Guiotti $^{4}$, Kamila Santos Tavares ${ }^{5}$, Luiza Oliveira Gomes ${ }^{6}$.

Resumo: Esse artigo buscou discutir os benefícios da cirurgia percutânea em comparação com a cirurgia cardíaca para correção da comunicação interatrial (CIA) ostium secundum. O trabalho trata-se de uma revisão bibliográfica a partir dos achados no campo da cirurgia cardíaca e uma análise dos riscos e vantagens apresentados pelo procedimento minimamente invasivo no tratamento do defeito do septo atrial (DSA), o qual apresenta-se como alternativa para os pacientes, independentemente da idade, por ser mais seguro para complicações de nível moderado, entregar melhora da capacidade funcional, de remodelamento cardíaco e melhor resultado estético quando comparado à cirurgia convencional. A cirurgia percutânea para correção da CIA depende de fatores como anatomia favorável e dimensionamento correto do dispositivo, são baixas as taxas de complicações graves relativas a esse procedimento, sendo a frequência de 1-5\%. Dessa forma, a cirurgia percutânea apresenta-se mais vantajosa para o tratamento da CIA ostium secundum quando comparada à abordagem cirúrgica cardíaca convencional.

Palavras-chave: Defeitos do septo atrial, Cirurgia torácica, Cardiopatias congênitas.

\begin{abstract}
This article aims to discuss the benefits of Percutaneous Coronary Intervention compared to cardiac surgery to correct ostium secundum Atrial Septal Defects. The study is a bibliographic review based on the findings in the field of cardiac surgery and an analysis of the risks and advantages presented by the minimally invasive surgical techniques in the treatment of atrial septal defect (ASD), which presents itself as an alternative for patients, regardless of age, for being safer for moderate level complications, delivering improved functional capacity, cardiac remodeling and better aesthetic results when compared to conventional surgery. Percutaneous intervention to correct ASD depends on factors such as favorable anatomy and correct sizing of the device, the rates of serious complications related to this procedure are low, with a frequency of 1 to $5 \%$. Thus, percutaneous surgery is more beneficial for the treatment of ASD ostium secundum when compared to the conventional cardiac surgical approach.
\end{abstract}

Key words: Atrial septal defect, Thoracic surgery, Congenital heart defects.

\footnotetext{
${ }^{1}$ Centro Universitário de Caratinga (UNEC), Caratinga - MG. *E-mail: amandarios17@hotmail.com

${ }^{2}$ Universidade de Vassouras (UV), Vassouras - RJ.

3 Universidade Vila Velha (UVV), Vila Velha - ES.

${ }^{4}$ Fundação Educacional do Município de Assis (FEMA), Assis - SP.

${ }^{5}$ Universidade do Oeste Paulista (UNOESTE), Presidente Prudente - SP.

${ }^{6}$ Universidade Federal de Juiz de Fora (UFJF), Juiz de Fora - MG.
} 
Resumen: Este artículo quiso discutir los beneficios de la cirugía percutânea em comparación a la cirugía cardiaca para el cierre de los defectos del tabique interatrial ostium secundum. Este trabajo trata de hacer un repaso bibliográfico a partir de publicaciones en el campo de la cirugía cardíaca y una análisis de los riesgos y ventajas del procedimento mínimamente invasivo para la corrección de los defectos del septo interatriales (DSA), lo que se presenta como una alternativa para los pacientes, independiente de su edad, ya que es un procedimiento seguro, con niveles moderados de complicaciones, y resultados de mejoras en la capacidad funcional, además, el resultado estético es ventajoso en comparación com la cirugía abierta. La cirugía percutánea de cierre de DSA depende de factores como la anatomia favorable y el dimensionamento correcto del dispositivo, son bajas las tasas de complicaciones graves relativas a este procedimiento, siendo la frecuencia de 1-5\%. Por lo tanto, la cirugía percutánea es más ventajosa para el tratamiento de DSA ostium secundum en comparación con el abordaje quirúrgico cardiaco convencional.

Palabras clave: Defectos del tabique interatrial, Cirugía torácica, Cardiopatías congénitas.

\section{INTRODUÇÃO}

A comunicação interatrial $(\mathrm{CIA})$ é um dos defeitos congênitos mais comuns na população, representando de $7 \%$ a $11 \%$ das doenças cardíacas congênitas (CHAMIÉ F, et al., 2020), e acometem o dobro de indivíduos do sexo feminino em relação ao masculino (BEHJATI-ARDAKANI M, et al., 2016). Existem, na literatura, quatro tipos de CIA: ostium secundum, ostium primum, tipo seio venoso e tipo seio coronário. Sendo o primeiro o tipo mais comum, representando $75 \%$ dos casos, e o único que permite tratamento percutâneo. Desse modo, será a comunicação interatrial do tipo ostium secundum (CIA OS), exclusivamente, retratada nesta revisão (BRASIL, 2018).

A CIA ostium secundum caracteriza-se por defeitos do septo interatrial (DSA) localizados na fossa oval. Em crianças, o DSA não possui sintomas específicos, o que está relacionado com o diagnóstico tardio, entretanto, com o decorrer da idade pode apresentar-se como uma intolerância ao exercício e pode manifestar sintomas como insuficiência cardíaca congestiva, taquiarritmias atriais, e hipertensão pulmonar, em adultos não tratados, está relacionada com uma baixa na expectativa de vida (TAKAYA Y, et al., 2015; GEVA T, et al., 2014).

O tratamento da CIA pode ocorrer por meio cirúrgico convencional, ou por intermédio de implante de dispositivo percutâneo. Ambos são eficazes para a correção, contudo, o tratamento cirúrgico é mais invasivo, pois se trata de uma toracotomia de peito aberto, em que é necessária uma circulação extracorpórea o que pode ocasionar efeitos pós-cirúrgicos como uma cicatriz esteticamente ruim e Síndrome de Resposta Inflamatória Sistêmica (SIRS) (OOI Y, et al., 2016; HOASHI T, et al., 2014).

Já o tratamento percutâneo é feito através de um cateter inserido na perna do paciente, portanto se apresenta como um tratamento minimamente invasivo, o que evita a necessidade de circulação extracorpórea, reduz a necessidade hospitalização, e possui rápida recuperação do paciente (TAKAYA Y, et al., 2015; HOASHI T, et al., 2014; RIBEIRO M, et al., 2013).

Até o momento a cirurgia percutânea é ofertada como tratamento apenas para a Comunicação Interatrial do tipo Ostium Ssecundum (CIA OS), portanto, está revisão tem como objetivo analisar os benefícios da cirurgia percutânea em comparação com a cirurgia cardíaca no tratamento da CIA OS.

\section{REVISÃO BIBLIOGRÁFICA}

\section{Anatomia/fisiologia da comunicação interatrial}

O coração é um órgão muscular oco dividido em quatro câmaras, duas superiores (átrios) e duas inferiores (ventrículos). $O$ átrio direito recebe o sangue venoso e se comunica com o ventrículo por meio da valva tricúspide. Após receber o sangue, os ventrículos se contraem para conduzi-lo por meio das artérias. Os átrios e ventrículos são divididos por uma parede muscular denominada septo interatrial e interventricular, respectivamente (BRASIL, 2018).

Durante a vida fetal é normal a existência de uma comunicação interatrial que permite a passagem do fluxo de sangue oxigenado proveniente do ducto venoso e do forame oval direto para o átrio esquerdo, uma 
vez que os pulmões ainda são incapazes de fornecer oxigênio. Após o nascimento ocorre o fechamento do forame oval devido ao aumento de pressão no átrio esquerdo. O defeito no fechamento dessa comunicação interatrial é uma cardiopatia congênita que permite a comunicação entre os átrios e a passagem de sangue do átrio esquerdo para o direito sobrecarregando as câmaras direitas (RIBEIRO MS, et al., 2017).

A CIA equipara-se de $7 \%$ a $11 \%$ de todos os defeitos cardíacos congênitos. Sua prevalência é de 0,53 por mil nascidos vivos. A CIA é classificada de acordo com sua localização anatômica no septo atrial (GEVA T, et al., 2014). A comunicação interatrial do tipo ostium secundum apresenta-se em 941 pacientes por 1 milhão de nascidos vivos, sendo frequente e ocorrendo predominantemente de forma aleatória. (RIBEIRO MS, 2017).

A comunicação interatrial produz shunt atrial da esquerda para a direita, visto que a pressão do átrio esquerdo é maior que a do átrio direito. Esse shunt ocasiona sobrecarga de volume do coração direito. Tal efeito pode passar despercebido na infância, mas algumas complicações, como arritmias e intolerância a exercícios, podem se manifestar no final da infância e na adolescência, havendo maior risco de doença vascular pulmonar obstrutiva (RAO PS E HARRIS AD, 2017). O fechamento espontâneo é de ocorrer nos primeiros anos de vida. Um estudo demonstrou que o maior número de fechamento espontâneo de defeitos do septo atrial ocorreram em pacientes com menos que 2 anos de idade. Nesse estudo, fechamento espontâneo ocorreu somente durante a infância, ocorrendo em $21,9 \%$ de todos os pacientes acompanhados (BEHJATI-ARDAKANI M, 2016).

No entanto, pacientes que permanecem com a doença devem realizar procedimentos de fechamento do defeito do septo atrial. Tais procedimentos podem ocorrer via cirurgia ou via fechamento percutâneo.

\section{Tratamento cirúrgico}

A cirurgia convencional é realizada por intermédio de uma esternotomia mediana ou uma incisão submamária direita, sendo aplicada anestesia geral. Há o canulamento da artéria aorta e da veia cava para colocar o paciente em circulação extracorpórea. O coração permanece parado durante o procedimento. Uma atriotomia direita é realizada para expor a CIA. O fechamento do defeito pode ser realizado através da aproximação das margens com sutura ou através do uso de um retalho de pericárdio, ou Dacron, cuja escolha é baseada no tamanho do defeito e na preferência do cirurgião. $O$ fechamento primário possibilita ruptura da sutura e abertura do defeito no futuro, sendo seu uso reservado para defeitos menores. Já o fechamento com retalho é utilizado para defeitos maiores e pode estar relacionado com shunt residual (RAO PS E HARRIS AD, 2017; BRASIL, 2018).

Os índices de sucesso da cirurgia convencional são altos, o que justifica sua permanência como padrão ouro de tratamento para CIA por décadas desde sua primeira realização em 1953 (KODAIRA M, et al., 2017). Entretanto, existem algumas desvantagens a ela relacionadas. Durante a cirurgia, a abertura e a circulação extracorpórea apresentam os riscos de infecção, hipoperfusão cerebral, lesões estéticas, efeitos psicológicos e déficit de aprendizado (BRASIL, 2018). Em um estudo realizado por Hoashi T, et al. (2014), o fechamento cirúrgico acarretou 3 infartos cerebrais pós cirurgia e em um índice de mortalidade de $0,32 \%$. Além disso, a necessidade de transfusão de sangue homólogo é outra desvantagem da cirurgia, podendo gerar infecções virais e respostas anafiláticas. No mesmo estudo, $12 \%$ dos pacientes que passaram por cirurgia, necessitaram de transfusão.

\section{Tratamento cirúrgico minimamente invasivo}

Cirurgias cardíacas realizadas sem esternotomia total são denominadas Cirurgias Minimamente invasivas para Correção de CIA. A cirurgia minimamente invasiva pode ser realizada pela técnica de mini-toracotomia direita, em que, com anestesia geral, é feita uma incisão de 6 a $8 \mathrm{~cm}$ no $4^{\circ}$ espaço intercostal ânterolateralmente. Há o uso de ecocardiograma transesofágico para auxílio durante o procedimento. Circulação extracorpórea e cardioplegia também estão presentes, semelhante à cirurgia convencional (KODAIRA M, et al., 2017).

Toracotomia anterolateral direita é realizada desde 1980. Antes da padronização do fechamento percutâneo para a CIA, mesmo as cirurgias minimamente invasivas eram mais bruscas e desagradáveis ao corpo e, portanto, não se detinha muita atenção à qual procedimento utilizar. Após a introdução do método percutâneo, a demanda por procedimentos mais esteticamente agradáveis aumentou, sendo a cirurgia 
minimamente invasiva uma escolha para aqueles que não podem ser submetidos ao fechamento percutâneo (HOASHI T, et al., 2014).

A cirurgia minimamente invasiva possui altas taxas de sucesso. Em um estudo de 2017, houve uma taxa de $100 \%$ de sucesso do procedimento. Nesse mesmo estudo, os autores puderam demonstrar 5 achados: fechamento percutâneo e cirurgia minimamente invasiva possuem altos índices de sucesso, o tempo de permanência no hospital foi menor no grupo de pessoas que realizaram fechamento percutâneo, a ocorrência de complicações maiores tendeu a ser maior no grupo que realizou a cirurgia, a ocorrência de complicações menores foi menor no grupo que realizou fechamento percutâneo e idade maior que 70 anos e cirurgia minimamente invasiva são fatores independentes para complicações (KODAIRA M et al., 2017).

Apesar do espaço operatório menor e de maior tempo de bombeamento, a cirurgia minimamente invasiva, em comparação com a cirurgia convencional, preserva a integridade esternal, possui melhor aspecto estético, reduz infecções de ferida, resulta em menor necessidade de analgesia e está associada com recuperações mais precoces (RASLAN S, et al., 2017).

As complicações relacionadas com a cirurgia minimamente invasiva são as mesmas da cirurgia convencional.

\section{Tratamento percutâneo}

Existem diversos dispositivos para o tratamento percutâneo da comunicação interatrial (CIA), por exemplo, Amplatzer, Figulla Flex II, Gore Helex, patch transcateter, entre outros (KAZMOUZ S, et, al., 2013). Todos os dispositivos portam uma malha autoexpansível composta principalmente de Nitinol (liga metálica de níquel e titânio), por meio das cinturas autocentráveis ocorre o mecanismo de oclusão com o preenchimento do orifício septal, e os discos acoplados em cada átrio após o implante formam uma barreira física ao fluxo sanguíneo. (RIBEIRO MS, et al., 2017).

Com o apoio de ecocardiograma transesofágico (ETE), o fechamento da CIA por implante de dispositivo percutâneo é realizado sob anestesia geral. Usando um cateter de dimensionamento, as propriedades anatômicas específicas dos defeitos do septo atrial (DSA) são determinadas, por exemplo, presença de uma borda adequada ao nível atrioventricular, localização, tamanho, entre outras. Para diferentes tipos de DSA, existem dispositivos específicos utilizados no fechamento, então, se o DSA for considerado adequado para oclusão percutânea baseado no diâmetro medido e em características morfológicas específicas, um dispositivo é escolhido. Em seguida, é utilizado um cateter maior para introduzir e liberar o oclusor, sob orientação por ETE e fluoroscopia. Quando uma posição adequada e estável em todo o defeito é alcançada, o dispositivo é liberado. Para a obtenção de um tempo de protrombina (alvo de 60-80 seg) o paciente é heparinizado por 24 horas. A alta do paciente acontece no dia seguinte, com a ingestão oral de antiagregante plaquetário por pelo menos seis meses (BRASIL, 2018).

Ao serem analisados os possíveis efeitos adversos, o fechamento percutâneo demonstrou-se ser mais seguro do que a cirurgia convencional para as complicações consideradas de nível moderado, notou-se uma redução de $73 \%$ em eventos adversos moderados a favor da intervenção. Além de excelentes resultados estéticos, não há necessidade de circulação extracorpórea (CEC), menos trauma e um menor tempo de internação (BRASIL, 2018).

Um estudo brasileiro obteve $100 \%$ de sucesso técnico no tratamento percutâneo da CIA, havendo somente 1 caso de complicação, em que ocorreu bloqueio atrioventricular total (BAVT). O dispositivo foi então retirado e o BAVT se resolveu em $36 \mathrm{~h}$. Após 6 meses, o paciente foi submetido novamente ao procedimento, tendo sucesso. Logo, conclui-se que é um procedimento factível em crianças (RIBEIRO MS, et al.,2013).

Um estudo envolvendo 244 pacientes com mais de 50 anos que realizaram o fechamento percutâneo avaliou os resultados depois de um maior tempo de acompanhamento. Os pacientes foram divididos em grupos de 50 a 59 anos, de 60 a 74 anos e de pessoas com mais de 75 anos. Os maiores achados foram que $90 \%$ dos pacientes com mais de 75 anos não tiveram causa de morte ou hospitalização relacionados com insuficiência cardíaca, ou Acidente Vascular Encefálico (AVE); os resultados a longo prazo não foram diferentes dos resultados dos outros grupos; A capacidade funcional e de remodelamento cardíaco melhoraram nos pacientes com mais de 75 anos, da mesma forma que nos outros grupos. Esses achados 
indicam a eficácia do procedimento e a viabilidade em idosos, visto que os resultados são favoráveis, com poucas complicações (TAKAYA Y, et al., 2015). Não parece haver limites de idade para a realização do procedimento.

Por consequência de uma anatomia desfavorável do DSA ou dimensionamento errado do dispositivo, possíveis complicações podem ocorrer. Os eventos mais comuns na fase de pós-procedimento são deslocamento do dispositivo, arritmias e embolismo. A taxa de complicações relativas ao procedimento é baixa. As complicações graves como mal posicionamento e cirurgia subsequente ocorrem em uma frequência de $1-5 \%$, embolização do dispositivo $0,4-4 \%$, acidente cerebrovascular $0,1-1,3 \%$, tamponamento cardíaco em 0,1\%, perfuração cardíaca em 0,03\% e endocardite em 0,03\% (BRASIL, 2018).

Tromboembolismo, erosão cardíaca, hipersensibilidade ao material do dispositivo implantado, enxaqueca, anomalias de condução, dano valvular, arritmias atriais e endocardite são complicações relatadas, mas ocorrem em poucos casos, podendo estar relacionadas a situações específicas de cada paciente (JALAL Z, et al., 2016).

\section{Tratamento cirúrgico versus tratamento percutâneo}

O defeito do septo atrial (ASD) pode ser solucionado por meio da cirurgia convencional, cirurgia cardíaca minimamente invasiva (MICS) e também, pela técnica percutânea. Sabe-se que, dos quatro tipos de CIA, apenas o defeito ostium secundum é suscetível de tratamento percutâneo de oclusão (RAO PS E HARRIS $A D, 2017)$.

Ao comparar o fechamento percutâneo com a MICS, o entendimento é que ambos os procedimentos possuem altas taxas de êxito, contudo é viável como primeira opção de recurso terapêuticoo fechamento percutâneo, quando o paciente apresenta anatomia apropriada. Além da alta taxa de eficácia, é preciso observar outros fatores que fazem o tratamento percutâneo ser preferível entre as MICS como, redução no tempo de internação e menor risco de complicações. (KODAIRA M, et al., 2017; RAO PS E HARRIS AD, 2017).

Além das MICS, a cirurgia convencional também é uma opção de oclusão da CIA, como seu índice de sucesso é elevado, ainda é uma opção de tratamento. É possível constatar que entre a cirurgia convencional e a minimamente invasiva, a MICS é a mais vantajosa, visto que preservar a integridade esternal, melhor resultado estético, reduz a infecções de feridas, melhor analgesia e ainda é associada a recuperação mais precoce (RASLAN S, et al., 2017; BRASIL, 2018).

A oclusão da CIA por implante percutâneo é uma alternativa segura e eficiente para o fechamento cirúrgico tradicional, com inúmeras vantagens, excelentes resultados estéticos, menor trauma, sem a obrigação de circulação extracorpórea (CEC), reduzido volume de sangue transfundido e ainda, diminuição do tempo de internação.

Embora possua suas vantagens não é um procedimento isento de riscos, as possíveis complicações podem derivar de uma anatomia desfavorável e dimensionamento errado do dispositivo. Após o procedimento, eventos mais comuns são o deslocamento do dispositivo, embolismo e arritmias. Logo, para a realização de um procedimento de qualidade e com redução de riscos, são necessários médicos habilitados. No Brasil existem problemas relacionados com a fila de espera em cirurgias cardíacas infantis e necessidade de ampliação ao acesso à cirurgia cardíaca infantil (BRASIL, 2018).

Além disso, um estudo mostrou que o risco de complicações é reduzido quando esses procedimentos percutâneos são realizados em hospitais cujo volume de casos é maior. Houve redução de $4,6 \%$ do risco em hospitais que performaram de 10 a 25 casos por ano, em relação aos hospitais que performam menos que 10 procedimentos. Há, ainda, uma redução de $2,1 \%$ do risco nos hospitais que realizaram mais que 25 procedimentos por ano, em relação aos que realizaram de 10 a 25 . Tais achados mostraram uma relação entre o volume anual de casos e a redução dos riscos, sugerindo uma recomendação de pelo menos 10 procedimentos ao ano, em coerência com os atuais guidelines (SINGH V, et al., 2015; KING III SB et al., 2007). 


\section{CONSIDERAÇÕES FINAIS}

A partir do exposto, conclui-se que o tratamento da CIA por intermédio do implante de dispositivo percutâneo é uma alternativa atraente à cirurgia, visto que é um procedimento minimamente invasivo que raramente apresenta efeitos adversos e que possibilita uma recuperação rápida do paciente. Além disso, não há limite de idade para a realização do implante de dispositivo, o que o torna uma opção viável para todas as faixas etárias. Entretanto, o procedimento não é isento de riscos, sendo de suma importância a atuação de médicos habilitados na sua execução. Ademais, é fundamental a elaboração de pesquisas que explorem o efeito a longo prazo do tratamento por meio do dispositivo percutâneo, na perspectiva de que esse procedimento seja considerado padrão ouro para o tratamento da CIA futuramente.

\section{REFERÊNCIAS}

1. RIBEIRO MS, et al. Estado Atual do Tratamento dos Defeitos do Septo Atrial. Revista da Sociedade de Cardiologia do Estado de São Paulo, 2017; 27(1): 39-48.

2. CHAMIÉ F, et al. Oclusão transcateter de comunicação interatrial do tipo seio venoso - uma nova opção terapêutica?. Journal of Transcatheter Interventions, 2020; 28 (1).

3. BEHJATI-ARDAKANI M, et al. The Clinical Course of Patients With Atrial Septal Defects. Iran J Pediatric, 2016; 26(4).

4. HOASHI T, et al. Management of ostium secundum atrial septal defect in the era of percutaneous trans-catheter device closure: 7-Year experience at a single institution. J Cardiol (2014).

5. GEVA T, et al. Atrial septal defects. The Lancet, 2014.

6. TAKAYA Y, et al. Long-Term Outcome After Transcatheter Closureof Atrial Septal Defect in Older Patients. Cardiovascular Interventions, 2015; 8 (4): 600 - 6.

7. OOI Y, et al. Transcatheter Versus Surgical Closure of Atrial Septal Defects in Children. Cardiovascular Interventions, 2016; 9 (1): $79-86$.

8. RASLAN S, et al. Outcome variables of right anterolateral mini thoracotomy versus complete

9. sternotomy in atrial septal defectclosure: A randomized controlledtrial. Journal of the Egyptian Society of CardioThoracic Surgery, 2017.

10. SINGH V, et al. Influence of Hospital Volume on Outcomes of Percutaneous Atrial Septal Defect and Patent Foramen Ovale Closure: A 10-Years US Perspective. The Society for Cardiovascular Angiography and Interventions, 2015; $85: 1073-1081$.

11. JALAL Z, et al. Long-term Complications After Transcatheter Atrial Septal Defect Closure: A Review of the Medical Literature. Canadian Journal of Cardiology, 2016.

12. KODAIRA M, et al. Comparison of Clinical Outcomes After Transcatheter vs.

13. Minimally Invasive Cardiac Surgery Closure for Atrial Septal Defect. Japanese Circulation Society, 2017.

14. KING SB, et al. Update of the clinical competence statement on cardiac interventional procedures: a report of the American College of Cardiology Foundation/American Heart Association/American College of Physicians Task Force on Clinical Competence and Training (writing Committee to Update the 1998 Clinical Competence Statement on Recommendations for the Assessment and Maintenance of Proficiency in Coronary Interventional Procedures). Journal of the American College of Cardiology, 2007; 50(1): 82-108.

15. RAO PS, HARRIS AD. Recent advances in managing septal defects: atrial septal defects. F1000 Research, 2017,6 : 2042.

16. BRASIL. Ministério da Saúde. Procedimento para fechamento percutâneo de comunicação interatrial septal com dispositivo intracardíaco. Brasília, 2018.

17. SOCHON E, et al. Transcatheter closure as an alternative and equivalent method to the surgical treatment of atrial septal defect in adults: Comparison of early and late results. Med Sci Monit, 2009; 15 (12): CR612-617.

18. CHEN Q, et al. Intraoperative device closure of atrial septal defects with inferior vena cava rim deficiency: A safe alternative to surgical repair. The Journal of Thoracic and Cardiovascular Surgery, 2011; 141(3): 631-636.

19. NYBOE C, et al. Long-term mortality in patients with atrial septal defect: a nationwide cohort-study. European Heart Journal, 2018; 39: 993-998.

20. VILLABLANCA PA, et al. Treatment Options for the Closure of Secundum Atrial Septal Defects: A Systematic Review and Meta-Analysis. International Journal of Cardiology, 2017.

21. WYSS Y, et al. Interventional Closure of Secundum Type Atrial Septal Defects in Infants Less Than 10 Kilograms: Indications and Procedural Outcome. Journal of Interventional Cardiology, 2016.

22. KONG D, et al. Three-Dimensional Echocardiography in the Evaluation of Right Ventricular Global and Regional Systolic Function in Patients with Atrial Septal Defectbefore and after Percutaneous Closure. Echocardiography, 2015; 33(4): 596-605.

23. NYBOE C, et al. Atrial fibrillation and stroke in adult patients with atrial septal defect and the long-term effect of closure. Heart, 2015; 101(9): 706-711.

24. AKULA VS, et al. Right Ventricle before and after Atrial Septal Defect Device Closure. Echocardiography, 2016; 33(9): 1381-1388.

25. BAYKAN AO, et al. Both Systemic and Pulmonary Artery Stiffness Predict Ventricular Functional Recovery after Successful Percutaneous Closure of Atrial Septal Defects in Adults. Congenit Heart Dis. 2016; (11):144-154.

26. ASKARI B, et al. Short-term outcomes after surgical versus transcatheter closure of atrial septal defects; a study from Iran. The Egyptian Heart Journal, 2018; 249-253. 